\title{
Early Pneumopericardium after Endoscopic Thoracic Sympathectomy
}

\author{
Mehmet Mutlu \\ Department of Anesthesiology and Reanimation, Okmeydani Education and Research Hospital, Sisli, Istanbul, Turkey
}

\begin{abstract}
Pneumopericardium is a condition characterised by the presence of air within the pericardium. We hereby present a case of a rare potentially life-threatening complication, which is, to our knowledge, the first report of the pneumopericardium after single port endoscopic thoracic sympathectomy (SPETS). A 25-year male patient with a history of bilateral palmar hyperhidrosis underwent SPETS. Routine chest X-ray at post-anesthesia care unit showed presence of diffuse subcutaneous emphysema, helping to establish the diagnosis of pneumopericardium with no symptoms after the surgery. Pneumopericardium should be considered among the possible complications of SPETS. Such complication may progress to a fatal event, if not recognised early and managed rapidly. Based on clinical experience, routine postoperative clinical follow-up protocols should be established and applied for each patient following SPETS.
\end{abstract}

Key Words: Palmar hyperhidrosis, Endoscopic thoracic sympathectomy, Pneumopericardium.

How to cite this article: Mutlu M. Early Pneumopericardium after Endoscopic Thoracic Sympathectomy. J Coll Physicians Surg Pak 2020; 30(09):980-982.

\section{INTRODUCTION}

Thoracic sympathectomy (TS) is performed in several pathologic conditions of autonomic nervous system such as palmar hyperhidrosis $(\mathrm{PH})$, characterised by excessive sweating of palms and increased sympathetic activity without involving thermoregulation. PHhassubstantial impactonaffected individuals' social relationships and quality of life. It usually occurs in the second and third decade of life and affects up to $2.8 \%$ of the American population. ${ }^{1}$ Mainstay medical therapies include antidepressants, anticholinergics, and botulinum toxin. ${ }^{1,2}$ Sympathectomy could be performed in cases where there is no response to medical treatment. ${ }^{1}$

TS was performed first by Hughes in 1942. Nowadays, sympathectomy is done as an outpatient surgery by cutting, burning, or clipping the sympathetic chain directly at the upper thoracic level. TS could also be performed endoscopically with theadvantage of shorter time, less cosmetic defect, reduced rate of complications and less pain due to single thoracoport method. ${ }^{3,4}$ All techniques involve Kuntz nerve ablation. Sympathicolysis for hyperhidrosis of facial, hands, and axillary regions is performed at the level of 2 , T2-T3, and T2-T4, respectively. ${ }^{2}$

Correspondence to: Dr. Mehmet Mutlu, Department of Anesthesiology and Reanimation, Okmeydani Education and Research Hospital, Sisli, Istanbul, Turkey

E-mail: drmmutlu@gmail.com

Received: April 11, 2019; Revised: October 02, 2019;

Accepted: October 22, 2019

DOI: https://doi.org/10.29271/jcpsp.2020.09.980
Many studies have been performed to improve outcomes, reduce operative costs, facilitate early discharge, and minimise complications with endoscopic TS (ETS). These include comparison of rigid versus flexible endoscopy, or general versus sedation anesthesia, assessment of surgical techniques like single-port endoscopic TS (SPETS), and even comparison of different types of sympathectomies, e.g via cauterisation or clamping. ${ }^{3,4}$ None of these studies reported a complication similar to what we hereby report. We present the case of a rare potentially life-threatening complication, which is, to our knowledge, the first report of the pneumopericardium after SPETS.

\section{CASE REPORT}

A 25-year male patient with ASA I was planned to undergo SPETS upon diagnosis of bilateral PH. After 3 minutes of induction of anesthesia, the patient was intubated with Robertshaw doublelumen tube (LRT). The site was confirmed by chest auscultation and flexible fiberoptic bronchoscope. Single lung ventilation was performed during surgery. During the operation, the patient remained stable without any hemodynamic instability, desaturation, increase in end-tidal $\mathrm{CO}_{2}$, or elevation of the lung plateau or peak pressures. The only problem occurred during port placement in the right hemithorax after the right anterior axillary line incision, where the port was placed with difficulty despite single lung ventilation. Afterwards, sympathetic chain dissection and ETS were performed. After adequate ventilation and lung expansion, the incision was closed without any chest tube placement. There were no surgical complication. The patient was extubated without any problem and was followed up in post-anesthesia care unit. In our hospital, all patients who underwent ETS are routinely checked with chest X-ray to avoid 
any life-threatening predictable complication, like pneumothorax or unpredictable uncommon complication such as pneumopericardium. In this patient, after routine physical examination, Hamman's sign was found with auscultation and there was subcutaneous emphysema on palpation. Control chest radiography revealed subcutaneous emphysema and pericardial air in the right hemithorax.

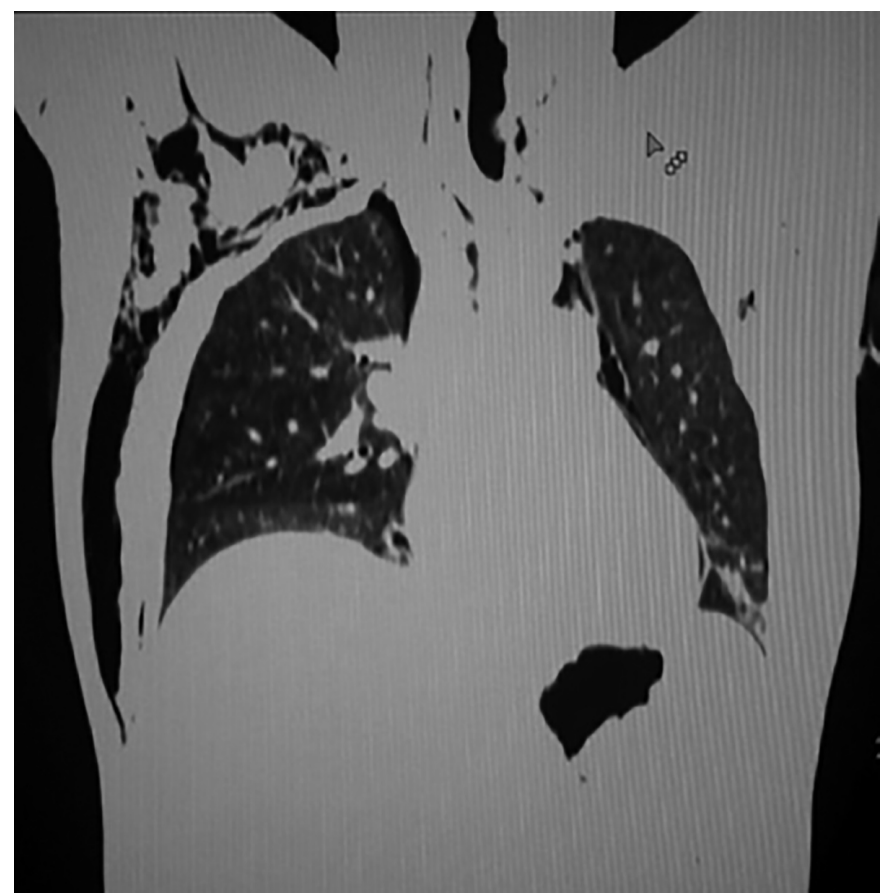

Figure 1: CT scan of thorox showing subcutaneous emphysema.

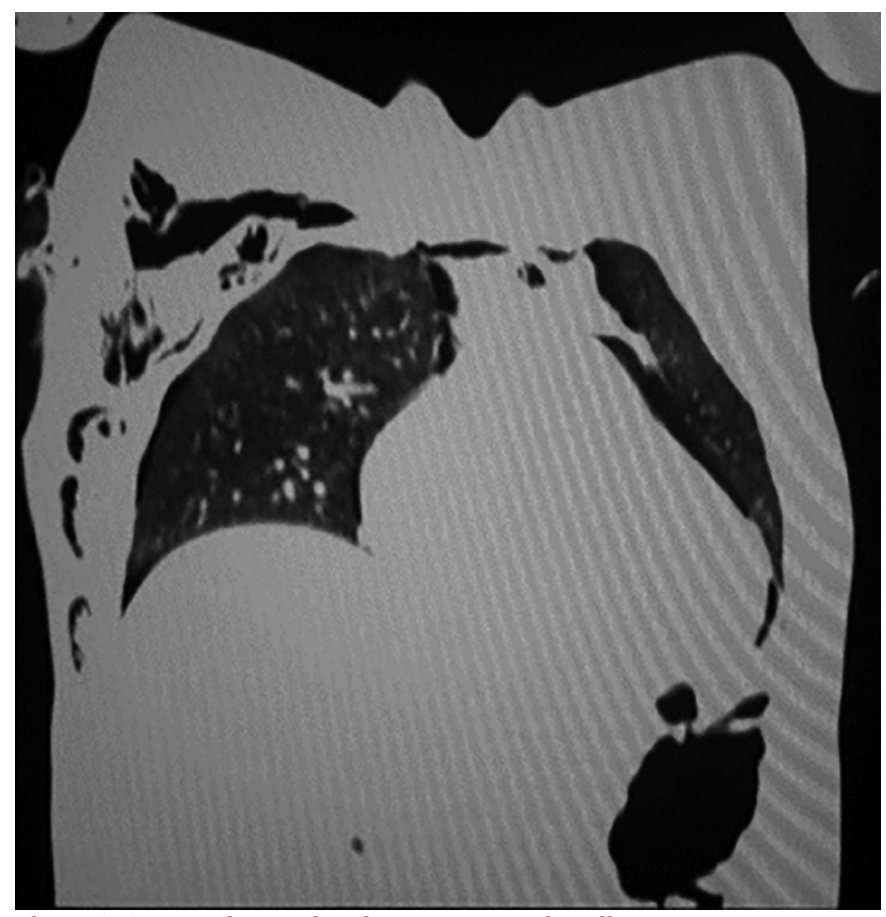

Figure 2: CTscan thorax showing pneumopericardium.

The patient's hemodynamic data and clinical status were stable, and his general status was good. Thorax CT was performed urgently. Thorax CT revealed diffuse subcutaneous emphysema, minimal mediastinal emphysema, and pneumopericardium. The patient was followed up postoperatively by chest roentgenograms as he was symptomless. Spontaneous improvement was observed in patient's clinical status and there was no need for any further surgical procedure (Figures 1 and 2).

\section{DISCUSSION}

ETS may be associated with surgical complications such as bleeding and infection. Another common complication (with a long-term incidence of 35-98\%) after TS is the compensatory hyperhidrosis, i.e. excessive sweating in the back, waist, groin, and legs of patients without preoperative sweating. ${ }^{1}$ It may relapse after one year of surgery with nerve regeneration and lysis. Horner's syndrome may also develop from cutting the sympathetic chain from the upper level. Bradycardia is another complication which can be observed after sympathectomy.

Pneumothorax, atelectasis, hemothorax, subcutaneousanesthesia with large or intercostal vessels injury, pain in the incision or dermatome area, chylothorax, and pneumopericardium are other rare complications due to thoracoscopy. Pneumopericardium is the presence of air in the pericardial area. It may develop due to blunt or penetrating thoracic trauma or iatrogenic reasons. Simple pneumopericardium is asymptomatic and does not cause any circulatory problems. However, it very rarely manifests as tension pneumopericardium, which causes severe cardiovascular insufficiency and circulatory disorder, requiring urgent surgical intervention. There is a reported case of sudden cardiac arrest due to pneumopericardium after trauma. $^{5}$

In cases of spontaneous pneumopericardium, alveolar pressure increases; and free air that progresses along the bronchovascular sheath after alveolar rupture, reaches the mediastinum. ${ }^{5}$ In our case, single lung ventilation had been performed and there was no alveolar rupture. The possible mechanism may be the dissection of the vascular sheath during sympathectomy and the development of pneumopericardium with the spread of air between the pericardial leaves. ${ }^{6}$ Pneumopericardium did not cause a circulatory problem and was simple in character. Usually associated with positive pressure ventilation, 37\% of these rare simple pneumopericardium cases were reported to progress to tension type, necessitating immediate surgical intervention..$^{7-10}$ This rate is close up to $70 \%$ in newborns with mechanical ventilation. Therefore, if mechanical ventilation is planned in patients with pneumopericardium, pericardial decompression is recommended before initiating ventilation. The underlying mechanism of this is the congenital pleuropericardial fistula, which works as a one-way valve.

These patients may also have symptoms such as chest pain, dyspnea, syncope, and upper quadrant pain. In the physical examination, each heart beat on auscultation is characterised by a crunching sound, called as the Hamman's sign. In addition, a 'bruit de moulin' murmur (mill-wheel shaped) and a pericardial rub can be heard on auscultation. The diagnosis is 
established with radiological findings. The air surrounding the heart is observed. The difference from pneumomediastinum is that there is no air above the upper border of the pericardium, and the air is displaced in the decubitus position. ${ }^{6}$ In our case, air was observed in the pericardium, and mediastinum as well as under the skin on thoracic CT, confirming the diagnosis radiologically. For the treatment, patients are monitored and followed up with series of chest X-rays. Spontaneous resolution is usually observed in patients who do not progress into tension pneumopericardium. During management of pneumopericardium, checking for tamponade symptoms is very critical as it could end up with a fatal outcome; therefore, it requires emergent intervention. ${ }^{8}$

Pneumopericardium presents a diagnostic challenge as shown in this case. Therefore, based on clinical experience, routine postoperative clinical and radiographical follow-up protocols should be established and applied to every patient following SPETS. A careful assessment of the chest radiography is crucial for diagnosis of pneumopericardium. Accurate evaluation of the radiologic signs of pneumomediastinum can help clinicians plan appropriate treatment in affected patients.

\section{PATIENT'S CONSENT:}

Patient's informed consent was obtained for publication of the case data.

\section{CONFLICT OF INTEREST:}

The author declared no conflict of interest.

\section{AUTHOR'S CONTRIBUTION:}

The author prepared this paper by himself without any help.

\section{REFERENCES}

1. Chwajol M, Barrenechea I, Chakraborty S, Lesser J, Connery C, Perin N. Impact of compensatory hyperhidrosis on patient satisfaction after endoscopic thoracic sympathectomy. Neurosurgery 2009; 64(3):511-8. discussion 518.

2. Heckmann M, Baumann C, Plewig G. Botulinum toxin A for axillary hyperhidrosis (excessive sweating). N Engl J Med 2001; 344(7):488-93.

3. Lin TS, Kuo SJ, Chou MC. Uniportal endoscopic thoracic sympathectomy for treatment of palmar and axillary hyperhidrosis: Analysis of 2000 cases. Neurosurgery 2002; 51(5 Suppl):S84-7.

4. Reisfeld R, Nguyen R, Pnini A. Endoscopic thoracic sympathectomy for hyperhidrosis: Experience with both cauterization and clamping methods. Surg Laparosc Endosc Percutan Tech 2002; 12(4):255-67.

5. Takahashi, K, Nagao K, Momokawa T, Maruyama A, Fujita $\mathrm{H}$, Ono S, et al. Two cases of idiopathic pneumopericardium. Kokyu To Junkan 1991; 39(1):95-7.

6. Zylak CM, Standen JR, Barnes GR, Zylak CJ. Pneumomediastinum revisited. Radiographics 2000; 20(4): 1043-57.

7. Haan JM, Scalea TM. Tension pneumopericardium: A case report and a review of the literature. Am Surg 2006; 72(4):330-1.

8. Gorecki PJ, Andrei VE, Schein M. Tension pneumopericardium in chest trauma. J Trauma 1999; 46(5):954-6.

9. Levin S, Maldonado I, Rehm C, Ross S, Weiss R. Cardiac tamponade without pericardial effusion after blunt chest trauma. Am Heart J 1996; 131(1):198-200.

10. Capizzi PJ, Martin M, Bannon MP. Tension pneumopericardium following blunt injury. J Trauma 1995; 39(4): 775-80. 